\title{
The role of Survivin as a biomarker and potential prognostic factor for breast cancer
}

\author{
DGlaucia Luciano da Veiga ${ }^{1}$ \\ Raissa Daniel Machado da Silva \\ (iD) Edimar Cristiano Pereira ${ }^{3}$ \\ (iD) Ligia Ajaime Azzalis ${ }^{3}$ \\ Deatriz da Costa Aguiar Alves ${ }^{1}$ \\ (iD) Flavia de Sousa Gehrke ${ }^{1,2}$ \\ (iD) Thaís Moura Gascón ${ }^{1}$ \\ Dernando Luiz Affonso Fonseca ${ }^{1,3}$
}

\begin{abstract}
1. Department fo Clinical Analysis - Medical School of ABC - FMABC - Santo André, SP, Brasil 2. Public Servant State Hospital - lamspe - São Paulo, SP, Brasil
\end{abstract} 3. Faculty of Pharmaceutical Sciences - Federal University of São Paulo - Unifesp, Diadema, SP, Brasil

http://dx.doi.org/10.1590/1806-9282.65.6.893

\section{SUMMARY}

Breast cancer $(B C)$ is one of the primary health problems worldwide. As the most common cancer in women in the world and in Brasil, behind only non-melanoma skin cancer, this neoplasm corresponds to approximately $28 \%$ of new cases per year in the country. BC also affects men, although the incidence corresponds to only $1 \%$ of total cases. Currently, most of the chemotherapeutic agents used in BC treatment are extremely toxic and cause long-term side effects. There is also a need to obtain earlier diagnoses, more accurate prognoses and make new therapies available that are more selective and effective in order to improve the current scenario. Therefore, this work sought to evaluate the importance of the biomarker survivin (Sur) in relation to BC, through the detailing of the role of Sur as a biomarker, the correlation between this protein and the prognosis of BC patients, and a summary of therapeutic strategies that target Sur for the development of new anticancer therapies.

KEYWORDS: Breast Neoplasms. Prognosis. Neoplasm Metastasis. Inhibitor of Apoptosis Proteins.

\section{INTRODUCTION}

Breast cancer (BC) is one of the primary health problems worldwide. As the most common cancer in women in the world and in Brasil, behind only non-melanoma skin cancer, this neoplasm corresponds to approximately $28 \%$ of new cases per year in the country. BC also affects men, although the incidence corresponds to only $1 \%$ of total cases ${ }^{1}$.

There are numerous types of BC, and currently, 15 types are known. Among the most common are: Ductal carcinoma in situ (DCIS), Lobular carcinoma in situ (LCIS), Invasive ductal carcinoma (IDC), Invasive Lobular Carcinoma (ILC), and Inflammatory Carcinoma of the Breast, which is an aggressive type of cancer, but rare. The incidence of breast cancer is not common before 35 years of age; from that period onward cases increase progressively, especially above the age of $50^{1-3}$.

According to the National Institute of Cancer (INCA) ${ }^{1}$ in Brasil, in 2016 the estimated number of new cases was 57,960 . The number of deaths caused

DATE OF SUBMISSION: 10-|an-2019

DATE OF ACCEPTANCE: 09-Feb-2019

CORRESPONDING AUTHOR: Glaucia Veiga

Principe de Gales Avenue, 821, Santo Andre, SP - Brasil - 09060-650

Tel: +55 11 4993-5488

E-mail: grlveiga@gmail.com 
by breast cancer reached 14,388 in 2013 , with 181 men and 14,206 women'. Despite the high number of deaths, it is known that the sooner the diagnosis is made, the better the chances of a good prognosis and successful treatment outcomes.

However, most chemotherapy agents currently used in the treatment of breast cancer are extremely toxic and cause long-term side effects, and there are also therapies that are no longer effective against certain types of BC. Faced with this situation, the demand for new therapies and antineoplastic drugs that are more specific and effective has grown in recent years ${ }^{4,5}$.

Lately, the focus has been on the development of genetic therapies to treat $\mathrm{BC}$, and one of the possibilities of gene therapy involves the use of survivin ${ }^{4}$. Survivin (Sur) is the smallest component of the inhibitor of apoptosis protein (IAP) family, and its importance is due to its specific expression in tumors that is unique among all human genetic products. Its expression is identified during embryonic and fetal development due to its role in controlling cell division, and it is also identified in several different types of human cancers because of its role in inhibiting apoptosis ${ }^{6}$. Apoptosis inhibition through Sur is linked to a negative prognosis and decreased survival in cancer patients ${ }^{7}$.

Given the relevance of the global health problem caused by breast cancer, the decreased effectiveness of existing treatments and the alarming number of people diagnosed with breast cancer each year, the main objective of this study was to identify the relevance of the role of Sur in the physiopathology of BC. Therefore, BC and the biomarker Sur will be considered; the role of Sur in the prognosis of $\mathrm{BC}$ and its therapeutic potential in cancer will also be addressed. Figure 1 shows a summary of the relationship between the pro-apoptotic pathway and Sur.

\section{METHODS}

The present work consists of carrying out research of an exploratory and a descriptive nature, which aims to relate the variables to the central analysis. The results will be presented qualitatively, taken from information collected from primary and secondary sources, and presented in the form of a bibliographic review.

The PUBMED and SCIELO databases were used, and a search with the terms "survivin" AND "breast
FIGURE 1. CLASSICAL INTRINSIC PATHWAY OF SURVIVIN (SUR) ACTION IN APOPTOSIS.

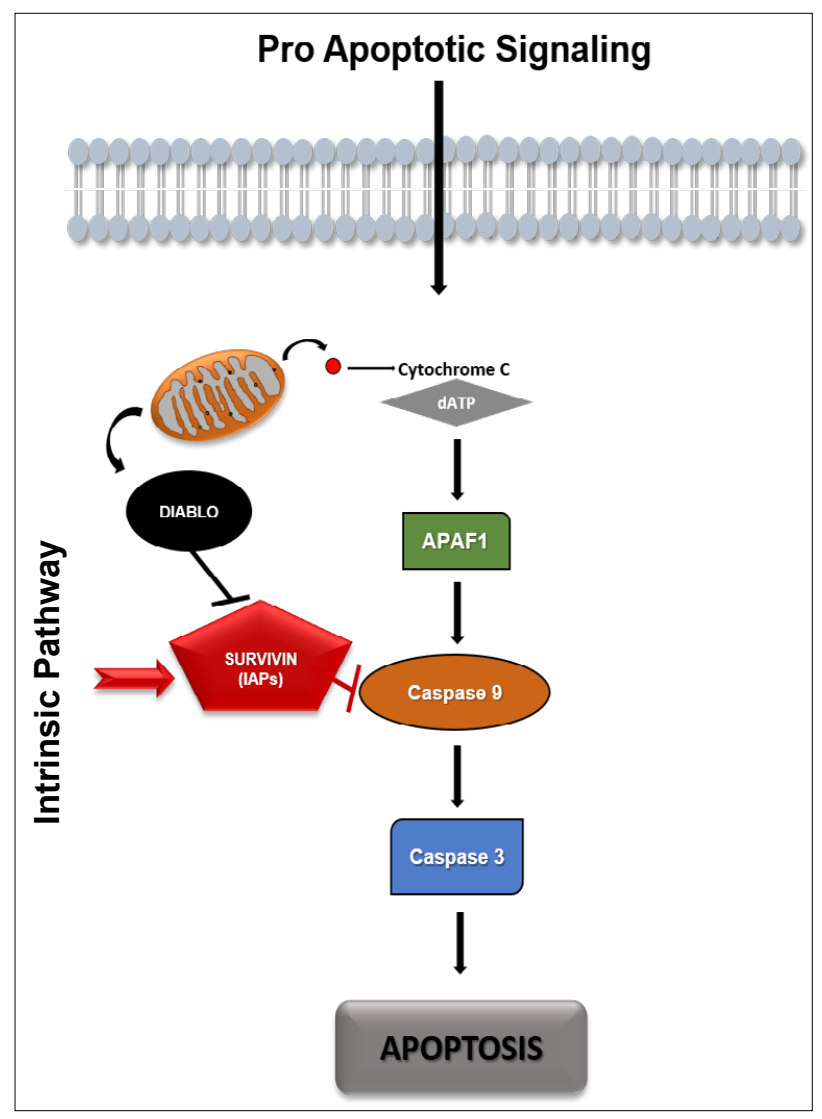

From a pro-apoptotic stimulus coming from the extracellular medium, cytochrome $\mathrm{C}$ is released from the mitochondria and interacts in the cytosol with the 2'-deoxyadenosine 5'-triphosphate (dATP) molecule, this interaction activates the apoptotic protease activating factor 1 (APAF1), which in turn activates caspase 9 and caspase 3 leading to apoptosis. The DIABLO or dehydrogenase-dependent activator of caspase (SMAC) is also released by the mitochondria, and its main functions include the inhibition of apoptosis inhibitory proteins (IAPs), such as Survivin (Sur). Survivin is known as the smallest of the IAPs and plays an important role in the apoptosis regulation through direct inhibition of caspase 9 .

cancer" AND "prognosis", and "survivin" AND “metastasis" was performed.

The inclusion criteria chosen for articles in this review were the presence of the terms above in the title or in the abstract of the articles; of these, articles that were published between the years 2012 and 2017, which were in English or Portuguese and fully available for analysis.

\section{RESULTS}

A total of 40 articles were obtained, of which 5 were outside the stipulated period, and 5 others did 
not address the theme proposed in this study and did not have Sur as the focus of study. Thus, only 30 articles remained to be analyzed (Figure 2).

\section{DISCUSSION}

$\mathrm{BC}$ is a malignant tumor resulting from the unbridled multiplication of abnormal cells ${ }^{1}$, resulting from some type of genetic mutation, that is installed in the mammary glands, usually starting in the mammary ducts (that connect the gland to the nipple) or in the breast lobules (milk-secreting parts). BC is classified as invasive and noninvasive. Invasive or infiltrative carcinoma is one that invades the breast tissues, and after growing, it infiltrates the skin and pectoral muscles; its malignant cells can migrate to the lymphatic circulation and reach the armpits and organs, such as the lungs, bones, brain, among others, a process called metastasis. Noninvasive carcinoma, also known as carcinoma in situ, is an initial tumor whose growth has not reached the basal membrane that separates the ducts and lobules of the breast from neighboring tissues ${ }^{8}$.

In the classification of $\mathrm{BC}, 15$ types are known, and the most common are: Ductal carcinoma in situ (DCIS), Lobular carcinoma in situ (LCIS), Invasive ductal carcinoma (IDC), Invasive Lobular Carcinoma (ILC) and Inflammatory Carcinoma of the Breast, which is an aggressive cancer, but rare. In addition to the specific types of $\mathrm{BC}$, there are also stages of cancer ranging from $O$ to IV, $O$ being a non-invasive type of cancer and IV indicating metastatic cancer ${ }^{2,3,9}$. According to Pavlidou et al. ${ }^{7}$, the risk factors related to the onset of breast cancer include genetic susceptibility, mutations in moderate-to high-penetration genes (BRCA1 / 2, PALB2, CHEK2, BRIP1, RAD50, NSB1 etc.), hormones associated with reproductive factors such as altered menstrual cycle, birth of firstborn child at an advanced age, use of hormonal therapies, alcohol consumption and type of diet, obesity, exposure to radiation and atypical hyperplasia of the mammary gland ${ }^{7}$.

According to INCA ${ }^{1}$ in Brasil (2016), the estimate of new cases was 57,960. The number of deaths caused by BC reached 14,388 in 2013, with 181 men and 14,206 women. The incidence of $\mathrm{BC}$ is not common before 35 years of age, and although the incidence is lower in young patients, the prognosis is worse in most cases, especially in patients under 40 years of age. After this period, cases increase pro-
FIGURE 2. REPRESENTATIVE SCHEME OF THE CHOICE OF ARTICLES.

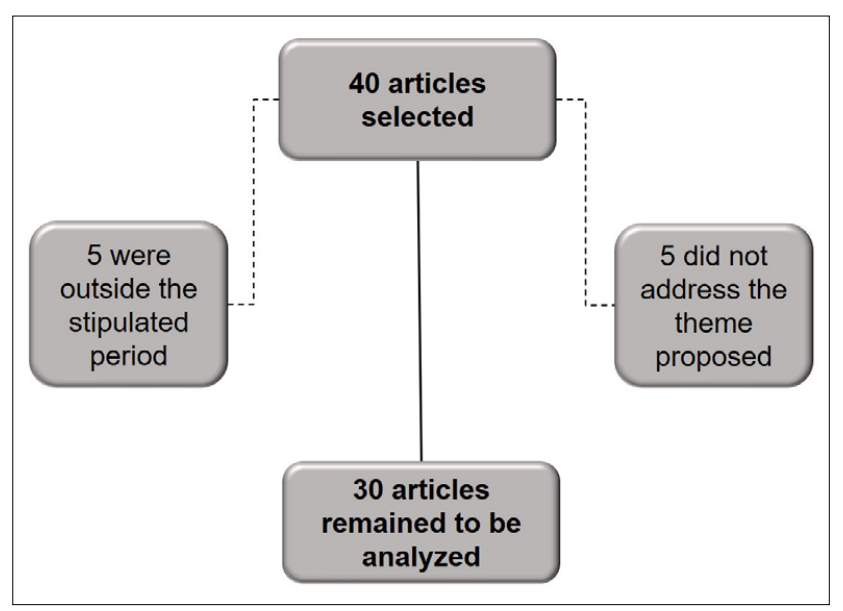

gressively, especially above 50 years of age ${ }^{1}$. However, it is known that the sooner the diagnosis is confirmed, the higher the chances of a good prognosis and success in the therapeutic course ${ }^{10}$.

The most common forms of early diagnosis of BC include breast examination performed by a physician to check for lumps in the breasts and armpits, mammography, breast ultrasound, breast tissue biopsy, and magnetic resonance imaging. After confirmation of the presence of breast carcinoma, additional tests and procedures are performed to determine the stage of cancer, such as a blood test, computed tomography scan (CT Scan), and positron emission tomography (PET Scan). Determining the stage of cancer generates a more correct prognosis and helps decide the best available treatments ${ }^{9}$.

The choice of appropriate BC treatments depends on the type of carcinoma, its stage, extent, size, and sensitivity of the malignant cells to hormones, as well as the overall health status of the patient. Most patients undergo breast cancer surgery (lumpectomy and/or mastectomy) and receive supplementary treatments before or after surgery, such as chemotherapy, hormone therapy or radiation ${ }^{9}$. However, $\mathrm{BC}$ presents a high risk of recurrence and metastasis. In addition, as is natural for most diseases, this type of cancer has evolved over time, increasing in complexity. Even if all of the above treatments are used, the prognosis of the disease remains poor. Hence the urgency to seek new therapies and more effective treatments.

Cancer biomarkers can also be called tumor markers or biological markers. According to Almei- 
da et al. ${ }^{11}$, tumor markers (or biological markers) are macromolecules present in the tumor, blood or other biological fluids and their presence and/or changes in their concentrations are related to the genesis and growth of neoplastic cells. In general, biomarkers are proteins or parts of proteins synthesized in response to cancer, both by the tumor itself and by the organism ${ }^{10,11}$.

Regarding $\mathrm{BC}$, the main biomarkers used in diagnostic or disease progression tests are BRCA 1 and $2^{12}$, CA 15-3, CA 27-29, Epidermal Growth Factor Receptor (EGFR) or HER1, EGFR2 or HER2, and estrogen receptors (ER) and progesterone receptors $(\mathrm{PR})$ present in tumors ${ }^{11}$. In recent years, researchers have been searching for new biomarkers in order to improve early diagnosis and also the sensitivity of breast cancer prognosis outcome, among these is the Sur protein.

Sur is a protein belonging to the family of Inhibitors of Apoptosis (IAP), whose main characteristic is related to the baculovirus IAP repeat (BIR) domains. Sur corresponds to the baculovirus IPA repeat containing five domains (BIRC5). This protein acts as an inhibitor of apoptosis and participates in the control of angiogenesis and cell proliferation. Its role in controlling angiogenesis and cell proliferation is identified primarily during embryonic and fetal development ${ }^{13}$. On the other hand, the protein plays a role in inhibiting apoptosis by its overexpression in adult and neoplastic tissues ${ }^{7}$, hence its importance as a new cancer biomarker. Recently, other functions related to Sur have been discovered, such as its participation in the control of autophagy and immune system infiltration, in addition to modulation of the tumor microenvironment ${ }^{6,7}$.

Khan et al. ${ }^{6}$ verified that the various functions performed by Sur appear to be related to where it is found. Sur can be found both in the nucleus, where it plays a role in regulating mitosis, and in the cell cytosol, where it modulates the inhibition of apoptosis. This molecule is also present in the extracellular environment in the form of exosomes, in which Sur is released from neoplastic cells to the tumor microenvironment through small vesicles attached to a small membrane ${ }^{14}$. It is believed that the presence of Sur in the extracellular environment can increase the aggressiveness of the tumor and also diminish or inhibit the effectiveness of cancer treatments ${ }^{6}$.

Kennedy et al. ${ }^{15}$ in 2003 confirmed the compartmental cellular division of Sur in histological ana- lyzes, in this study tumor cells were collected from 293 cases of primary breast cancer. In this analysis, 176 tumors showed positive staining for Sur versus 117 that did not. Among the Sur positive tumors, 139 expressed the protein in the nuclear region; 91 showed staining only in the cell nucleus and 48 in both the nucleus and the cytoplasm. Another $37 \mathrm{tu}-$ mors showed the presence of survivin in the cytoplasm only ${ }^{15}$.

Like many gene transcription products, Sur features pairing variants in addition to the so-called wild-type Sur ${ }^{7}$. According to Khan et al. ${ }^{16}$, wild-type Sur has anti-apoptotic properties. The Sur-2B variant, in which a 69 bp exon was introduced, has a pro-apoptotic nature. Sur- $\Delta \mathrm{Ex} 3$ is characterized by the exclusion of exon 3 and has anti-apoptotic activity. On the other hand, a part of intron 3 was included in Sur-3B that also has anti-apoptotic activity ${ }^{\mathbf{1 6}}$.

The Sur a-2a variant, which is the shortest transcribed Sur, and contains the inclusion of a region of $197 \mathrm{bp}$ at the 3' end of intron 2 and also has a pro-apoptotic function ${ }^{17}$. In the same line of study, Pavlidou et al. ${ }^{7}$ present two other variants: Sur-2B +32 in which a sequence of 32 nucleotides in intron 2 was added to the sequence $2 \mathrm{~B}$, and Sur-image (SI) which consists of a final product of part of the gene (345 bp), a part of the image gene for eye cancer (155 bp) added to another insertion of $7 \mathrm{bp}$.

Sur is involved in tumorigenesis through various mechanisms, including inhibition of apoptosis, regulation of cytokinesis and cell cycle progression, and involvement in a variety of pathways such as p53, Wnt, hypoxia, TGF, and Notch signaling pathways ${ }^{16}$. However, the major aspect of Sur and its pairing variants is its role in the inhibition of apoptosis. The process of programmed cell death is important both in carcinogenesis and in the treatment of cancer. In mammals, thus occurs via two routes, the intrinsic pathway and the extrinsic pathway of apoptosis. Both pathways are controlled by caspases, and interactions with Sur occur with caspase-3, caspase-7, and caspase-9. Thus the inhibition of apoptosis via Sur ${ }^{12}$.

Knowing the relevance of Sur in the carcinogenic process, it is necessary to understand its role as a $\mathrm{BC}$ biomarker and how its concentrations can be measured. Khan et al. ${ }^{16}$ collected sera from 40 BC patients and then compared the results against 10 control patients who were free of the disease five years post-treatment. Samples were analyzed for the presence of Sur and its pairing variants and exosomes. 
In BC patients, levels of Sur were significantly higher than levels in the control patient samples. The amount of Sur and Sur- $\Delta \mathrm{Ex} 3$ were the same in the evaluated samples. On the other hand, the Sur-2B variant showed differential expression in the different stages of $\mathrm{BC}$ in the patients ${ }^{17}$. The latter result emphasizes the growing importance of the use of Sur as an early biomarker and the possibility of using its levels for prognostic determination.

Despite the numerous treatments and diagnostic tests available for BC, rates of disease recurrence and mortality still remain alarmingly high due to poor prognosis as well as delay in diagnosing the disease. BC prognosis takes into account the type of tumor, the stage of the disease, the age of the patient, the general state of health and therapies the patient had previously undergone ${ }^{16}$.

When the patient's prognosis is discussed, the terms survival rate and relative survival rate are used by physicians in order to assist in better understanding the prognosis itself. The survival rate is calculated based on the percentage of patients living at least five years after the cancer diagnosis and after receiving treatment therapies, without having a recurrence of the disease or metastasis. On the other hand, the relative survival rate also takes into account the cancer stage of the patient. Patients with breast cancer stage 0 or $\mathrm{I}$, in general, have a relative survival rate close to $100 \%$. For patients in stages II and III, the rate drops to $93 \%$ and $72 \%$, respectively. Stage IV has the worst relative survival rate of only $22 \%$. However, each is a particular case and rates are only estimates and may or may not prove accurate ${ }^{14}$.

In most cases, the earlier $\mathrm{BC}$ is diagnosed, the better the patient's prognosis, hence the importance of biological biomarkers such as Sur. As Sur is rarely expressed in healthy adult tissues and was identified in most cancers, including $\mathrm{BC}^{6}$, using it as a biomarker also to obtain prognoses could prove to be of extreme value. In this review, we considered articles that discussed the role of Sur and its transcript variants in the prognosis of breast cancer, both negative and positive.

According to Khan et al. ${ }^{16}$, supra-regulation of the Sur is found in some types of cancers, including breast cancer, and it is possible that it is associated with an advanced stage, as well as poor prognosis and low survival rates.

The Sur found in the nucleus of the cell can be both an indicator of the degree of neoplasia and as- sociated with a proliferative phenotype, besides correlating with the stage of the tumor and its histological type. In addition to nuclear Sur, the increased RNA expression of this protein characterizes an independent prognostic marker, coupled with the expression of Sur transcript variants ${ }^{6}$.

A study conducted by Lee et al. ${ }^{18}$ describes the relationship between the expression of Sur and the poor prognosis of BC. In this study, five different cancer cell lines were evaluated, and the highest expression of Sur was verified in the nucleus of the cell (57.27\% of the cases) with a decreased expression in the cytoplasm. In addition, the MDA-MB-231 strain, whose cells are potentially more metastatic than cells from other strains, showed a higher expression of predominantly nuclear Sur, since the presence of the marker in the cytoplasm of this cell line was lower ${ }^{18}$.

Another important finding of the study refers to the presence of nuclear Sur in cases in which factors related to tumor progression were identified, such as tumors larger than $2 \mathrm{~cm}$, Tumor, Node, Metastasis (TNM) staging system from II to III and metastasis in the lymph nodes. It was verified that tumors larger than $2 \mathrm{~cm}$ associated with lymph node metastasis were related to low survival rate and progression-free survival (PFS). In addition, more advanced TNM was positively correlated with worse PFS. Therefore, it could be inferred that the expression of nuclear Sur is associated with a negative prognosis for $\mathrm{BC}$ patients ${ }^{18}$.

Even though a correlation was identified between nuclear Sur expression and a negative prognosis for breast neoplasia, the study by Lee et al. ${ }^{18}$ failed to correlate the protein pairing variants with the prognosis. However, this correlation was described in a review of the literature carried out by Pavlidou et al. ${ }^{7}$, in which the Sur transcript variants were evaluated according to the clinicopathological characteristics of $\mathrm{BC}$ patients and their survival rate.

The Sur-3B variant was confirmed to be anti-apoptotic, which corroborates previous observations of its elevated expression in breast tumors with mutations in p53, and was also associated with patients who presented worse PFS. Sur- $\Delta \mathrm{Ex} 3$ expression has also been identified in this type of tumor. Some studies have correlated the presence of this variant with the increase of the histological grade of the tumor, mainly in ER-negative tumors. On the other hand, Sur-2a was expressed in low-grade non-invasive tumors, 
probably due to its pro-apoptotic nature. However, a comparative study between these four studies elucidated the role of Sur-2a and its association with a worse prognosis for BC patients ${ }^{7}$.

Although one of the studies revealed that wildtype Sur, Sur- $\Delta \mathrm{Ex} 3$, and Sur-2B are not associated with the prognosis of breast carcinoma, other articles confirmed that these variants, together with survivin-3B and survivin-2A were linked to a worse prognosis and progression-free survival. Another study demonstrated that the expression of wild-type survivin-2A and survivin-3B indicates an even worse prognosis. Additionally, there was confirmation that the interaction between the survivin variants might alter the patient's prognosis ${ }^{7}$.

Specifically regarding the Sur-2B type, an inverse relationship was determined regarding the amount of Sur-2B in patients whose lymph nodes were already compromised due to primary breast neoplasia. This inverse correlation, coupled with the expression ratio between Sur-2B/wild-type Sur and estrogen receptors, suggests that Sur-2B may play an antagonizing role in wild-type apoptotic inhibition and may be used to predict patient survival since it is a possible marker of tumor aggressiveness ${ }^{7}$.

Khan et al. ${ }^{16}$ identified the location of Sur and its variants in exosomes of $\mathrm{BC}$ patients and the expression of the proteins in these vesicles mirrored the expression patterns in neoplastic cells, suggesting the possibility of using them as biomarkers. In the analyzed samples Sur-2B was related to tumor aggressiveness. This variant was found mainly in primary tumors at the early stage of the disease, whereas in higher-grade tumors Sur-2B was expressed in much lower amounts or absent, as well as in more far-reaching metastases. It was concluded, therefore, that Sur-2B can be used for early diagnosis and serves as a positive prognosis for breast cancer ${ }^{16}$.

Finally, it could be inferred that to better determine the prognosis of a patient with $\mathrm{BC}$ it is imperative to detect the presence of Sur and its variants both in neoplastic cells and in their exosomes, in addition to considering the interaction between these proteins. Once identified, expectations are that it will be possible to distinguish patients with low-grade neoplasia who do not need more advanced and expensive treatments from patients who are at higher risk of death and need a more sensitive diagnosis so that they could be offered more appropriate and aggressive treatments ${ }^{17}$. Only then can the use of
Sur be validated as a diagnostic and prognostic biomarker and possibly as a target for the development of new therapies.

Pavlidou et al. ${ }^{7}$ described some ways of treating Sur and its isoforms as possible anti-cancer therapy targets, which have been confirmed and updated in the study by Chen et al. ${ }^{17}$. At first, it was thought that Sur could not become a new target due to the structural properties that presented an obstacle to this end. However, indirect mechanisms have been proposed as strategies for the use of this protein to increase the cellular response to apoptosis and to inhibit tumor growth, which are inhibition of its transcription, inhibition of post-translational Sur, vaccines based on Sur and gene therapy that introduces suppressor mutants which act on this protein ${ }^{7,17}$.

Initially, the strategy of the inhibition of the Sur transcription was used, as were RNA interference experiments, which can reduce the production of these proteins and confirm the fact that they are crucial for the survival of the tumor cells ${ }^{7}$.

RNA-based therapies involve the use of small interfering RNA (siRNA), antisense oligonucleotides, microRNA (miRNA) and ribozymes. An example can be cited involving Sur's signaling transcriptional target, HA-CD44, which was inhibited by siRNA, resulting in its decreased expression and the significant loss in the degree of breast neoplastic cell invasion. The miRNAs play the role of oncogenes or tumor suppressors, and the miR-203 group is a direct target in the case of triple-negative cancer cells. Silencing or deletion of this group has been proven to suppress tumors in this type of cancer. Sur antisense oligonucleotides have the function of suppressing the expression of this protein; for this, they must reach the target cells through chemically synthesized molecules or vectors, such as YM155 and LY2181308 agents that can inhibit the expression of all Sur isoforms. Ribozymes are small RNA molecules that break target RNA via endonucleolytic activity. More recent examples such as CUA110 (RZ7) and c (RZ1) have resulted in decreased Sur levels and increased caspase-9-dependent apoptosis; however, in the study of melanoma cells ${ }^{7,17}$.

Regarding Sur inhibition at the post-translational level, we can cite cyclin-dependent kinase (CDK) inhibitors and heat shock proteins (HSP), especially HSP90 which forms complex with Sur. CDK inhibition by flavopiridol and purvalanol A prevents mitotic phosphorylation of Sur at Thr34 and accelerates 
its degradation, resulting in decreased expression of this protein and the induction of apoptosis ${ }^{19}$.

Inhibition of HSP90, which functions as a molecular chaperone in protein conformation and generates preventive compounds when complexed to Sur, has been evaluated with the Shepherdin cell antagonist. This antagonist competes with Sur by binding to HSP90 and induces apoptosis through independent and caspase-dependent mechanisms. Regarding anticancer activity, Shepherdin also proved to be highly selective and is, therefore, a potential target for this type of therapy, since its toxicity in normal fibroblasts was found to be low, and no other side effects were observed in normal cells ${ }^{7,17}$.

Knowing that Sur also functions as a tumor-associated antigen ${ }^{7}$, the evaluation of the immune response against this protein through $\mathrm{T}$ lymphocyte recognition presents a new strategy to develop new therapies ${ }^{17}$. During a search for tumor-associated antigens, a spontaneous immunogenic response to the presence of Sur was identified via CD8+ lymphocytes. Based on this information, some vaccines began to be developed, and the suppression of tumor growth was demonstrated in some types of cancers without causing significant toxicity. Despite the high cost of vaccine development, several studies focusing on vaccines against Sur have already moved on to Phase II clinical trials. The fourth strategy currently employed for the use of Sur as a target in anti-cancer treatment is gene-based therapy. Within this strategy, there are two more promising approaches to inhibit this protein. The first of these is based on the activation of apoptosis in tumor cells transfected with Sur-containing vectors and the second is the expression of cytotoxic genes mediated by Sur promoters in tumor cells. The proposed positive aspect in the use of the gene-editing strategy is that this approach causes cell death of neoplastic cells associated with minimal or even no toxic effects on healthy cells ${ }^{17}$.

Other uses for Sur as a target for the treatment of $\mathrm{BC}$ include the association between radiotherapy and Sur, chemotherapy and Sur and improved chemotherapy through the inhibition of its isoforms, such as treatment with 5-fluoracil+epirubicin+cyclophosphamide and the combination of docetaxel+epirubicin (Tax-Epi) guided by measurements of the levels of Sur and its variants ${ }^{7}$.

Chen et al. ${ }^{17}$ verified that the use of siRNA was considered the most effective for Sur inhibition in relation to RNA interference molecules. On the oth- er hand, Wang et al. ${ }^{20}$ used the eukaryotic expression vector pIRES2-EGFP-Survivin siRNA to transfer siRNA to the MCF-7 breast cancer cell line, given the high expression of this protein in this lineage. It was concluded that the level of Sur was significantly lower in cells transfected with siRNA compared to the control group, which in turn resulted in decreased MCF-7 proliferation, migration and invasiveness, as well as an increase in the number of apoptotic cells ${ }^{20}$.

The use of siRNA for Sur was also combined with chemotherapeutic drugs to increase apoptosis and reverse drug resistance in MCF-7 mammary carcinoma cells, as detailed in the study conducted by Dong et al. ${ }^{21}$. It was concluded that the siRNA effectively inhibited the Sur expression and increased the degree of cell death induction already caused by paclitaxel and epirubicin, that is, the combination of therapies conferred MCF-7 cells increased sensitivity to chemotherapeutic drugs and cellular apoptosis ${ }^{15}$.

Other studies have focused on the development of vectors to transfer siRNA to Sur in BC cells. As an example, the polyethylene glycol-modified chitosan nanoparticle vector (PEG-CS) has been synthesized and the results show that PEG-CS is a safe and efficient vector in reducing tumor growth and in the prevention of metastasis ${ }^{16}$. A second vector that succeeded in the transfer of siRNA was the nanodiamond NDCONH(CH2)2NH-VDGR. This transporter showed an inhibitory effect on MCF-7 cell proliferation and Sur gene silencing ${ }^{14}$. Both transporters have been suggested as positive additions for use in the treatment of breast carcinoma targeting Sur.

Three studies found in this review discuss how the Sur infra-regulation pathway can be affected. The first one deals with how the use of eugenol, a natural phenolic compound found in honey and essential oils, can trigger the death of $\mathrm{BC}$ cells through the down-regulation of the E2F1 transcription factor and its anti-apoptotic target Sur. The down-regulation of E2F1 reduced the levels of Sur and increased the cytotoxic effect of eugenol on the target cells ${ }^{4}$. The second article discusses the inhibitor and inducer of autophagy SAHA currently being investigated for use in patients with BC. According to this study, the infraregulation of Sur is part of the autophagic role of SAHA, since the inducer causes a decrease in Sur gene expression, induces acetylation of the protein and also decreases its stability ${ }^{15}$. The last study involves the use of the miR-204 microRNA that tar- 
gets Janus kinase 2 (JAK2). However, this study verified that in addition to inhibiting JAK2 kinase, which suppressed cell viability and induced apoptosis of neoplastic breast cells, the increase in programmed cell death was also caused by inhibition of the Sur pathway ${ }^{22}$.

Concerning the Sur inhibition, one study evaluated that the bovine lactoferrin protein (bLf), both in iron-free form and saturated with the metal, was classified as an inhibitor of Sur expression and a modulator of the apoptotic process in BC cells ${ }^{23}$. The last study deals with the Sur inhibitor YM155, whose inhibition of gene transcription induced caspase-7 activation-dependent autophagy and DNA damage-dependent autophagy in neoplastic breast cells ${ }^{24}$.

It is inferred, therefore, that although Sur is not an enzyme or a cell surface molecule ${ }^{17}$ and it presents structural limitations, it is possible to make use of the several indirect strategies described so that this protein is included as a new target of $\mathrm{BC}$ treatments. Research on Sur continues with the objective of finding new strategies and ways to add the use of this protein to existing treatments and increase the final therapeutic effect ${ }^{5,7}$.

\section{CONCLUSION}

The high incidence of $\mathrm{BC}$ added to the still high mortality caused by this disease stimulates the scientific community to look for new diagnostic methods and develop new therapies to improve the current scenario. Early diagnosis of BC saves millions of lives each year, and an accurate prognosis helps in determining more effective treatments for each patient.

Since biomarkers have played a relevant role to the topics as mentioned earlier, the evaluation of Sur as a biomarker and a prognostic factor of $\mathrm{BC}$ brings to light new and interesting possibilities for the treatment of mammary carcinoma.

The articles used in this review showed that Sur is highlighted among the new biomarkers of cancer and especially for BC. As a prognostic factor, several authors differ as to the positive or negative representation that the expression of the protein and its variants confer on the patient's health, but studies are still being performed in order to reach a more accurate determination. However, there is no doubt that researchers around the world are committed to increasing the possibility and feasibility of using Sur as the target of new anticancer therapies.

Since this work used limiting criteria, even though its use made the production of this paper possible, it would be interesting to expand the research of bibliographic references, since there is still much content on the subject to be explored. Since BC is a disease with a high impact on world health, continuing research on the Sur biomarker is essential.

\author{
Funding statement \\ Not applicable. \\ Conflicts of interest \\ The authors declare no conflicts of interest.
}

\begin{abstract}
RESUMO
O câncer de mama (CM) é um dos principais problemas de saúde em todo o mundo. Como o câncer mais comum em mulheres no mundo e no Brasil, precedido apenas pelo câncer de pele não melanoma, essa neoplasia corresponde a aproximadamente 28\% dos novos casos por ano no país. O CM também afeta homens, embora a incidência corresponda a apenas $7 \%$ do total de casos. Atualmente, a maioria dos agentes quimioterápicos utilizados no tratamento do CM são extremamente tóxicos e causam efeitos colaterais a longo prazo. Há também a necessidade de se obterem diagnósticos mais precoces, prognósticos mais precisos e disponibilizar novas terapias seletivas e efetivas, a fim de melhorar o cenário atual. Portanto, este trabalho buscou avaliar a importância do biomarcador Survivina (Sur) em relação ao CM, por meio do detalhamento do papel do Sur como biomarcador, da correlação entre essa proteína com o prognóstico de pacientes com CM e de um resumo do tratamento terapêutico e das estratégias que visam utilizar a Sur para o desenvolvimento de novas terapias anticâncer.
\end{abstract}

PalaVRas-ChaVe: Neoplasias da mama. Prognóstico. Metástase neoplásica. Proteínas inibidoras de apoptose. 


\section{REFERENCES}

1. INCA. Instituto Nacional do Câncer José Alencar Gomes da Silva [homepage of internet]. Tipos de câncer: câncer de mama. [cited 2017 Oct 23]. Available from: http://www2.inca.gov.br/wps/wcm/connect/tiposdecancer/site/home/mama

2. Breastcancer.org. [homepage of internet]. Types of breast cancer. [cited 2017 Oct 23]. Available from: http://www.breastcancer.org/symptoms/ types

3. Instituto Oncoguia. Instituto Oncoguia. [homepage of internet]. Quais são os tipos de câncer de mama? [cited 2017 Oct 23]. Available from: http:// www.oncoguia.org.br/conteudo/quais-sao-os-tipos-de-cancer-de-ma$\mathrm{ma} / 3951 / 669 /$

4. Al-Sharif I, Remmal A, Aboussekhra A. Eugenol triggers apoptosis in breast cancer cells through E2F1/survivin down-regulation. BMC Cancer. 2013;13:600.

5. Wang $H$, Ye YF. Effect of survivin siRNA on biological behaviour of breast cancer MCF7 cells. Asian Pac J Trop Med. 2015;8(3):225-8.

6. Khan S, Ferguson Bennit H, Asuncion Valenzuela MM, Turay D, Diaz Osterman C), Moyron RB, et al. Localization and upregulation of survivin in cancer health disparities: a clinical perspective. Biologics. 2015;9:57-67.

7. Pavlidou $A$, Kroupis $C$, Dimas $K$. Association of survivin splice variants with prognosis and treatment of breast cancer. World J Clin Oncol. 2014;5(5):883-94.

8. Sociedade Brasileira de Mastologia. [homepage of internet] [cited 2017 Nov 11]. Available from: http://www.sbmastologia.com.br/index. php?option=com_content $\&$ view=article\&id=909: diagnostico-do-cancer-de-mama\&catid=115\&ltemid=707

9. Mayoclinic. Diseases and Conditions - Mayo Clinic. Diagnosing breast cancer. [cited 2017 Nov 11]. Available from: https://www.mayoclinic.org/ diseases-conditions/breast-cancer/diagnosis-treatment/drc-20352475

10. Kuniyoshi RK, Gehrke FS, Alves BC, Vilas-Bôas V, Coló AE, Sousa N, et al. Gene profiling and circulating tumor cells as biomarker to prognostic of patients with locoregional breast cancer. Tumour Biol. 2015;36(10):8075-

11. Almeida IRC, Pedrosa NL, Leite IB, Fleming TRP, Carvalho VH, Cardoso AAA. Marcadores tumorais: revisão de literatura. Rev Bras Cancerol. 2007:53(3):305-16

12. Wooster R, Bignell G, Lancaster J, Swift S, Seal S, Mangion J, et al. Identification of the breast cancer susceptibility gene BRCA2. Nature. 1995;378(6559):789-92
13. Pennati M, Campbell AJ, Curto M, Binda M, Cheng Y, Wang LZ, et al. Potentiation of paclitaxel-induced apoptosis by the novel cyclin-dependent kinase inhibitor NU6140: a possible role for survivin down-regulation. Mol Cancer Ther. 2005;4(9):1328-37.

14. Khan S, Jutzy JM, Aspe JR, McGregor DW, Neidigh JW, Wall NR. Survivin is released from cancer cells via exosomes. Apoptosis. 2003;16(1):1-12.

15. Kennedy S, O'Driscoll L, Purcell R, Fitz-Simons N, McDermott EW, Hill $A D$, et al. Prognostic importance of survivin in breast cancer. $\mathrm{Br}$ | Cancer. 2003;88(7):1077-83

16. Khan S, Bennit HF, Turay D, Perez M, Mirshahidi S, Yuan Y, et al. Early diagnostic value of survivin and its alternative splice variants in breast cancer. BMC Cancer. 2014;14:176.

17. Chen X, Duan N, Zhang C, Zhang W. Survivin and tumorigenesis: molecular mechanisms and therapeutic strategies. J Cancer. 2016;7(3):314-23.

18. Lee JY, Kuo CW, Tsai SL, Cheng SM, Chen SH, Chan HH, et al. Inhibition of HDAC3- and HDAC6-promoted survivin expression plays an important role in SAHA-induced autophagy and viability reduction in breast cancer cells. Front Pharmacol. 2016;7:81.

19. Al-Khalaf HH, Aboussekhra A. Survivin expression increases during aging and enhances the resistance of aged human fibroblasts to genotoxic stress. Age (Dordr). 2013;35(3):549-62.

20. Wang X, Qiu W, Zhang G, Xu S, Gao Q, Yang Z. MicroRNA-204 targets JAK2 in breast cancer and induces cell apoptosis through the STAT3/BCl2/survivin pathway. Int | Clin Exp Pathol. 2015;8(5):5017-25.

21. Dong $H$, Yao L, Bi W, Wang F, Song W, Lv Y. Combination of survivin siRNA with neoadjuvant chemotherapy enhances apoptosis and reverses drug resistance in breast cancer MCF-7 cells. J Cancer Res Ther. 2015;11(4):717-22

22. Li S, Wang L, Meng Y, Chang Y, Xu I, Zhang Q. Increased levels of LAPTM4B, VEGF and survivin are correlated with tumor progression and poor prognosis in breast cancer patients. Oncotarget. 2017;8(25):4128293.

23. Sun $P$, Huang $W$, Jin $M$, Wang $O$, Fan B, Kang L, et al. Chitosan-based nanoparticles for survivin targeted siRNA delivery in breast tumor therapy and preventing its metastasis. Int J Nanomedicine. 2016;11:4931-45.

24. Bi Y, Zhang Y, Cui C, Ren L, Jiang X. Gene-silencing effects of anti-survivin siRNA delivered by RGDV-functionalized nanodiamond carrier in the breast carcinoma cell line MCF-7. Int | Nanomedicine. 2016;11:5771-87. 